\title{
Inappropriate Antibiotic Utilization: Outpatient Prescription Review of a Regional Secondary Hospital in Kedah, Malaysia
}

\author{
Guo-Hong Tan ${ }^{1}$, Qiau-Wei Low ${ }^{1}$, Huey-Chyi Lim ${ }^{1}$,Hui-Koon Seah ${ }^{1}$, Huan-Keat Chan ${ }^{2 *}$ \\ ${ }^{1}$ Pharmacy Department, Hospital Sik, 08220 Sik, Kedah, MALAYSIA \\ ${ }^{2}$ Clinical Research Centre, Hospital Sultanah Bahiyah, 05460 Alor Setar, Kedah, MALAYSIA
}

\begin{abstract}
Received: 4 August 2017;
Accepted: 27 September 2017

*Correspondence to:

Mr. Huan-Keat Chan, MSc,

Clinical Research Centre, Hospital Sultanah Bahiyah, 05460 Alor Setar, Kedah, Malaysia. Email: huankeat123@yahoo.com

Copyright: (C) the author(s),publisher and licensee Indian Academy of Pharmacists. This is an openaccess article distributed under the terms of the Creative Commons Attribution Non-Commercial License, which permits unrestricted non-commercial use, distribution, and reproduction in any medium, provided the original work is properly cited.
\end{abstract}

Publishing Partner : EManuscript [www.emanuscript.in]

\begin{abstract}
Background: The National Antibiotic Guideline (NAG) was introduced in Malaysia in 2014, and has since become the guidance for antibiotic prescribing, especially in public health centers where infectious disease specialists, local antibiotic guidelines and clinical microbiology services are not readily available. This study was designed to assess the patterns of antibiotic use for a range of diseases in the outpatient department of a regional secondary hospital, and the level of adherence to the NAG among the prescribers. Methods: A cross-sectional study was undertaken at the Sik Hospital, Kedah. All oral antibiotic-containing prescriptions of adult outpatients dispensed during $1^{\text {st }}$ May and $31^{\text {st }}$ July 2016 were reviewed. The information on each prescription, including age, diagnosis, and the type and dosage of the antibiotic prescribed, was recorded on an electronic data collection sheet. Results: Of 8,312 prescriptions screened, $662(8 \%)$ contained at least one oral antibiotic. Inappropriate selection of antibiotics was found in approximately $40 \%$ of the prescriptions with clear diagnoses, the majority $(79.2 \%)$ of which involved the use of erythromycin ethylsuccinate for upper respiratory tract infections (URTIs). Besides, by comparing the defined daily dose (DDD) of each antibiotic with the maximum DDD as recommended in the NAG, inappropriate dosage and treatment duration were found in five antibiotics, particularly those prescribed for the treatment of URTIs and urinary tract infections. Conclusion: As antimicrobial resistance has become a major concern worldwide, the findings warrant immediate attention and a well-planned approach to improve the antibiotic prescribing practice among the secondary hospitals in Malaysia.

Key words: Anti-Bacterial Agents, Drug Resistance, Inappropriate Prescribing, Malaysia, Outpatients.
\end{abstract}

\section{INTRODUCTION}

Selection of medications without consideration for safety and efficacy, and failure to utilize medications in line with standard treatment protocols remain the major health problems in developing countries. ${ }^{[1]}$ Irrational use of antibiotics is especially of concern in light of the rapid development of antimicrobial resistance, ${ }^{[2]}$ which has not only led to the rising healthcare costs, but has also increased mortality, morbidity and health facility utilization. ${ }^{[3]}$ In addition, ineffective treatment for bacterial infections could highly affect the outcomes of numerous surgical procedures and cancer chemotherapy. ${ }^{[4]}$ Although antimicrobial stewardship programs (ASPs) have long been introduced to clinical practice worldwide, ${ }^{[5]}$ 
overuse and misuse of antibiotics were still frequently reported over the past five years. ${ }^{[1-6-9]}$

In Malaysia, the Ministry of Health has launched the protocol of the ASP in 2014 to promote rational use of antibiotics among the public health facilities. ${ }^{[10]}$ Furthermore, the National Antibiotic Guideline (NAG) was introduced following the nationwide program, ${ }^{[11]}$ and has become the guidance for physicians in antibiotic prescribing, particularly among primary and secondary health centers where infectious disease specialists, local antibiotic guidelines and clinical microbiology services were not readily available. However, to date, the majority of the existing studies on the use of antibiotics for outpatients in Malaysia have been conducted in primary health centers, focusing predominantly on upper respiratory tract infections (URTIs). ${ }^{[12-14]}$ Therefore, this study aimed to assess the patterns of antibiotic use for a range of diseases in outpatients, and the level of adherence to the NAG among the prescribers in a public secondary hospital.

\section{METHODS}

This cross-sectional study was undertaken at the Sik Hospital, a 93-bed secondary medical center in Kedah State, Malaysia, with five medical officers providing the outpatient medical services for a population of approximately 65,000. Prior to data collection, the study proposal was registered with the National Medical Research Register, Malaysia (NMRR-15-912-25332), and was approved by the Medical Research Ethics Committee, Malaysia.

All oral antibiotic-containing prescriptions of adult outpatients (12 years of age or above), which were dispensed in the pharmacy unit during $1^{\text {st }}$ May and $31^{\text {st }}$ July 2016, were retained for review by the investigators (pharmacists). A prescription was excluded if (i) the patient had received an intravenous loading dose of antibiotic in the ward, (ii) it contained multiple diagnoses of infections, or (iii) the diagnosis was not specified or illegible. After the eligibility was confirmed, the information on each prescription, including patient's identification number, age, diagnosis, and the type and dosage of the antibiotic prescribed, was recorded on an electronic data collection sheet.

Data management and analysis were conducted using the Microsoft Excel 2010 (Microsoft, Washington). The evaluation of antibiotic use focused on two aspects: (i) drug selection, and (ii) dosage and treatment duration.
First, the appropriateness of each antibiotic selected for a specific diagnosis was determined based on the recommendations in the NAG. ${ }^{[11]}$ Subsequently, the appropriateness of dosage and treatment duration was confirmed using the WHO Anatomical Therapeutic Chemical/ Defined Daily Dose (ATC/ DDD) method, which has also been widely used in previous studies. [15,16] The average DDD of each antibiotic used for a specific diagnosis was calculated using the WHO ATC/DDD formula (DDD = number of dose units of a defined strength $\mathrm{X}$ conversion factor) ${ }^{[15-17]}$ and it was then compared with the maximum DDD, which was calculated using the same formula based on the maximum daily dosage and treatment duration indicated by the NAG. ${ }^{[1]}$

\section{RESULTS}

Over the three-month study period, the pharmacy unit dispensed a total of 8,312 prescriptions, $662(8 \%)$ of which contained at least one oral antibiotic. However, 58 prescriptions were excluded from further analysis as the diagnoses were not specified $(7.4 \%)$ or illegible $(1.1 \%)$. Of 605 prescriptions included, antibiotics were most commonly used for URTIs (47.8\%), followed by skin and soft tissue infections (SSTIs) $(23.6 \%)$, urinary tract infections (UTIs) (13.6\%), community-acquired pneumonia (CAP) (6.9\%), leptospirosis $(2.1 \%)$, and ocular infections $(1.8 \%)$. The most frequently prescribed antibiotic was erythromycin ethylsuccinate $(34.4 \%)$, followed by cloxacillin (15.4\%), and amoxicillin/ clavulanic acid (11.6\%) (Table 1). All prescriptions contained a single antibiotic, except for 18 (3.1\%) with the combination of amoxicillin/ clavulanic acid and azithromycin for the treatment of CAP.

Inappropriate selection of antibiotics was found in more than one-third (39.7\%) of the prescriptions (Table 1). Of all these cases, a vast majority (79.2\%) of the patients received erythromycin ethylsuccinate for URTIs, while $17.9 \%$ of patients received ampicillin/ sulbactam for SSTIs (15.4\%), UTIs $(2.1 \%)$ and URTIs $(0.4 \%)$. Besides, inappropriate use of cefuroxime $(1.7 \%)$ and ampicillin $(1.3 \%)$ for CAP was identified. Furthermore, although the drug selection could be justified in most cases, inappropriate dosage and treatment duration was still found in five antibiotics: cefuroxime, doxycycline, cefalexin, phenoxymethylpenicillin and amoxicillin (Table 2). In particular, the doses of cefuroxime in 12 UTI cases (500mg per dose) and doxycycline in a URTI case (200mg per dose) were found to have doubled the recommended doses. Moreover, cefalexin and phenoxymethylpenicillin were prescribed as 
Tan et al:: Inappropriate antibiotic Use in a Malaysian secondary hospital

\begin{tabular}{|c|c|c|c|c|}
\hline Antibiotic class & Name of antibiotic & ATC code & $\begin{array}{l}\text { Number of } \\
\text { prescriptions }(\% \text { of } \\
\text { total) }\end{array}$ & $\begin{array}{l}\text { No. of prescriptions with } \\
\text { inappropriate antibiotic (\% of } \\
\text { total) }\end{array}$ \\
\hline \multirow[t]{5}{*}{ Penicillins } & Cloxacillin & J01CF02 & $93(15.4)$ & $0(0)$ \\
\hline & Amoxicillin/ clavulanic acid & J01CR02 & $70(11.6)$ * & $0(0)$ \\
\hline & Amoxicillin & J01CA04 & $58(9.6)$ & $3(1.3)$ \\
\hline & Ampicillin/ sulbactam & J01CR01 & $45(7.4)$ & $43(17.9)$ \\
\hline & Phenoxymethylpenicillin & J01CE02 & $9(1.5)$ & $0(0)$ \\
\hline \multirow[t]{2}{*}{ Macrolides } & Erythromycin ethylsuccinate & J01FA01 & $208(34.4)$ & $190(79.2)$ \\
\hline & Azithromycin & J01FA10 & $27(4.5)$ & $0(0)$ \\
\hline \multirow[t]{2}{*}{ Cephalosporins } & Cefuroxime & J01DC02 & $44(7.3)$ & $4(1.7)$ \\
\hline & Cefalexin & J01DB01 & $34(5.6)$ & $0(0)$ \\
\hline \multirow[t]{2}{*}{ Fluoroquinolones } & Ciprofloxacin & J01MA02 & $5(0.8)$ & $0(0)$ \\
\hline & Ofloxacin & J01MA01 & $1(0.2)$ & $0(0)$ \\
\hline Tetracycline & Doxycycline & J01AA02 & $21(3.5)$ & $0(0)$ \\
\hline Sulfonamide & Sulfamethoxazole/ trimethoprim & J01EE01 & $6(1)$ & $0(0)$ \\
\hline $\begin{array}{l}\text { Imidazole } \\
\text { derivative }\end{array}$ & Metronidazole & J01XD01 & $2(0.3)$ & $0(0)$ \\
\hline Total (\%) & & & $605(100)$ & $240(100)$ \\
\hline
\end{tabular}

\begin{tabular}{|c|c|c|c|}
\hline Diagnosis & Antibiotic & Average DDD & Maximum DDD \\
\hline \multirow[t]{6}{*}{ URTIs } & Doxycycline & 18.7 & 14 \\
\hline & Amoxicillin & 9.9 & 15 \\
\hline & Amoxicillin/ clavulanic acid & 7 & 10.5 \\
\hline & Cefuroxime & 7 & 14 \\
\hline & Phenoxymethylpenicillin & 6.5 & 5 \\
\hline & Azithromycin & 5 & 11.7 \\
\hline \multirow[t]{7}{*}{ SSTIS } & Doxycycline & 19 & 30 \\
\hline & Amoxicillin & 10.5 & 10.5 \\
\hline & Amoxicillin/ clavulanic acid & 7 & 15 \\
\hline & Cloxacillin & 6.8 & 10 \\
\hline & Cefalexin & 5.3 & 7 \\
\hline & Azithromycin & 3.3 & 3.3 \\
\hline & Erythromycin ethylsuccinate & 2.7 & 12 \\
\hline \multirow[t]{3}{*}{ UTIs } & Cefuroxime & 9.7 & 7 \\
\hline & Amoxicillin/ clavulanic acid & 7 & 21 \\
\hline & Cephalexin & 4.9 & 3.5 \\
\hline \multirow[t]{4}{*}{ CAP } & Amoxicillin/ clavulanic acid & 6.8 & 10.5 \\
\hline & Azithromycin & 5 & 5 \\
\hline & Erythromycin ethylsuccinate & 3.1 & 5.6 \\
\hline & Ampicillin/ sulbactam & 1.8 & 1.8 \\
\hline Leptospirosis & Doxycycline & 16.3 & 14 \\
\hline \multirow[t]{2}{*}{ Ocular infections } & Amoxicillin & 8.1 & 7.5 \\
\hline & Cloxacillin & 6.7 & 10 \\
\hline
\end{tabular}


three- and four-time-daily instead of twice-daily regimens in 32 UTI and 4 URTI cases, respectively. Apart from that, doxycycline and amoxicillin were, respectively, prescribed for seven instead of five days in five leptospirosis and one ocular infection cases. Nevertheless, none of the patients were found to be under dosed with antibiotics.

\section{DISCUSSION}

This is, to the investigator's knowledge, the first study to review the antibiotic use in a Malaysian regional secondary hospital following the launch of the NAG in 2014. Unlike previous studies focusing primarily on the primary care, ${ }^{[12-14]}$ this study was undertaken in the outpatient unit of a hospital setting, where a broader range of antibiotics, including extended-spectrum penicillins, azithromycin and cefuroxime, were used. Besides, instead of reflecting the prescribing patterns of antibiotics for a specific infection, ${ }^{[13-14]}$ it highlights a high degree of inappropriate antibiotic use for multiple diagnoses, which warrants immediate attention and a well-planned approach to improve the overall antibiotic prescribing practice.

Several key messages have emerged from this study. First, notwithstanding the WHO's recommendation of $30 \%$ for antibiotic use in an outpatient setting, ${ }^{[18]}$ antibiotics were present in only $8 \%$ of the prescriptions. The finding is consistent with that of a recent nationwide study, which demonstrated that the antibiotic prescribing rate among the Malaysian public health clinics was approximately $7 \%{ }^{[12]}$ Nevertheless, it is noteworthy that almost half of the patients in this study were prescribed an antibiotic for URTIs, which have been proven to be commonly of viral origin and self-limiting. ${ }^{[19]}$ Hence, despite the relatively low prescribing rate, there is a need to review the rationale behind the use of each antibiotic, particularly in URTIs.

Furthermore, the prescribers were apparently unaware of the fact that erythromycin ethylsuccinate, which contributed to approximately $80 \%$ of the prescriptions with inappropriate drug selection, has long been removed from the NAG as the first-line treatment for URTIs due to the resistance developed by Streptococci. ${ }^{[11-14]}$ Aside from that, inappropriate dosing was common, particularly in the treatment of UTIs, whereby cefuroxime and cefalexin were given a daily dose higher than the recommended doses in a total of 44 cases. Therefore, an effort should be made to increase the awareness of the prescribers about the recommendations in the NAG.
However, a number of study limitations were acknowledged. First, this is a single-center study, and thus the findings may not be representative of other public secondary hospitals in Malaysia. Besides, approximately $10 \%$ of the prescriptions containing antibiotics were excluded from further analysis due to unclear or illegible diagnoses. Additionally, the diagnoses of patients were determined based solely on what were written on the prescriptions. Future studies should therefore be multicenter in design, and further investigate the clinical reasoning of the antibiotic use.

\section{CONCLUSION}

This study confirms that inappropriate use of antibiotics was common in a Malaysian public secondary hospital. Underdosing did not appear to be a major concern; nonetheless, inappropriate drug selection and overdosing were found to have occurred frequently. A high level of non-adherence to NAG was identified, and thus a systematic approach to improve the overall antibiotic prescribing practice is warranted.

\section{ACKNOWLEDGEMENT}

We wish to thank the Director General of Health, Malaysia for his permission to publish the findings from this study. Besides, all the staffs from the Sik Hospital, who have helped us in acquiring the data, are acknowledged.

\section{SOURCES OF SUPPORT}

This research received no specific grant from any funding agency in the public, commercial, or not-forprofit sectors.

\section{CONFLICT OF INTEREST}

No conflicts of interest.

\section{ABBREVIATION USED}

NAG: National Antibiotic Guidelines. DDD: Defined daily dose. ATC: Anatomical Therapeutic Chemical. CAP: Community-acquired pneumonia. SSTI: Skin and soft tissue infection. URTI: Upper respiratory infection. UTI: urinary tract infection.

\section{REFERENCES}


Tan et al:: Inappropriate antibiotic Use in a Malaysian secondary hospital

1. Mao W, Vu H, Xie Z, Chen W, Tang S. Systematic review on irrational use of medicines in China and Vietnam. PLoS ONE. 2015;10(3):e0117710.

2. Ventola $C L$. The antibiotic resistance crisis: part 1: causes and threats. $P$ T. 2015;40(4):277-83.

3. Cosgrove SE. The relationship between antimicrobial resistance and patient outcomes: mortality, length of hospital stay, and health care costs. Clin Infect Dis. 2006;42 Suppl 2:S82-9.

4. LaxminaraSik R, Duse A, Wattal C, Zaidi AK, Wertheim HF, Sumpradit N, et al. Antibiotic resistance-the need for global solutions. Lancet Infect Dis. 2013;13(12):1057-98.

5. Leuthner KD, Doern GV. Antimicrobial stewardship programs. J Clin Microbiol. 2013;51(12):3916-20.

6. Hopkins $\mathrm{CJ}$. Inpatient antibiotic consumption in a regional secondary hospital in New Zealand. Intern Med J. 2014;44(2):185-90.

7. Priyendu A, Patel I, Nagappa AN. Antibiotic resistance: A growing concern. J Pharm Pract Community Med. 2015;1(1):2-4

8. Ahmad A, Patel I. Schedule H1: Is it a solution to curve antimicrobial misuse in India? Ann Med Health Sci Res. 2013;3(Suppl 1):S55-6.

9. Sun Q, Dyar OJ, Zhao L, Tomson G, Nilsson LE, Grape M, et al. Overuse of antibiotics for the common cold - attitudes and behaviors among doctors in rural areas of Shandong Province, China. BMC Pharmacol Toxicol. 2015;16:6

10. Pharmaceutical Services Division. Protocol on antimicrobial stewardship program in healthcare facilities. 1st ed, Ministry of Health Malaysia, Kuala Lumpur, 2014.

11. Pharmaceutical Services Division. National Antibiotic Guideline 2014. Available at: http://www.pharmacy.gov.my/v2/en. [Accessed November 15, 2016].

12. Ab Rahman N, Teng CL, Sivasampu S. Antibiotic prescribing in public and private practice: a cross-sectional study in primary care clinics in Malaysia.
BMC Infect Dis. 2016;16:208.

13. Teng CL, Achike FI, Phua KL, Norhayati Y, Nurjahan MI, Nor AH, et al. General and URTI-specific antibiotic prescription rates in a Malaysian primary care setting. Int J Antimicrob Agents. 2004;24(5):496-501.

14. Rezal RS, Hassali MA, Alrasheedy AA, Saleem F, Yusof FA, Kamal M, et al. Prescribing patterns for upper respiratory tract infections: a prescriptionreview of primary care practice in Kedah, Malaysia, and the implications. Expert Rev Anti Infect Ther. 2015;13(12):1547-56.

15. Bajis S, Van den Bergh R, De Bruycker M, Mahama G, Van Overloop C, Satyanarayana $\mathrm{S}$, et al. Antibiotic use in a district hospital in Kabul, Afghanistan: are we overprescribing? Public Health Action. 2014;4(4):25964

16. Hutchinson JM, Patrick DM, Marra F, Ng H, Bowie WR, Heule L, et al Measurement of antibiotic consumption: A practical guide to the use of the Anatomical Therapeutic Chemical classification and Definied Daily Dose system methodology in Canada. Can J Infect Dis. 2004;15(1):29-35.

17. WHO Collaborating Centre for Drug Statistics Methodology. ATC/DDD index. Available at: https://www.whocc.no/atc_ddd_index/.

18. WHO. Using indicators to measure country pharmaceutical situations: fact book on WHO Level I and Level II monitoring indicators. Available at: http:// www.who.int/medicines/publications/WHOTCM2006.2A.pdf.

19. Dekker AR, Verheij TJ, van der Velden AW. Inappropriate antibiotic prescription for respiratory tract indications: most prominent in adult patients. Fam Pract. 2015;32(4):401-7.

Cite this article as: Tan GH, Low QW, Lim HC, Seah HO, Chan HK. Inappropriate Antibiotic Utilization: Outpatient Prescription Review of a Regional Secondary Hospital in Kedah, Malaysia. J Pharm Pract Community Med. 2017;3(4):215-9. 